\title{
The Use of Smartphones for Clinical Communication on Internal Medicine Wards
}

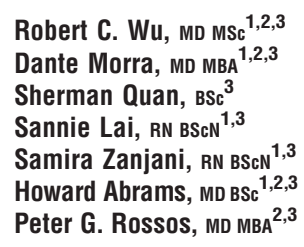

Robert C. Wu, MD Msc ${ }^{1,2,3}$

Dante Morra, MD MBA ${ }^{1,2,3}$

Sherman Quan, BSc ${ }^{3}$

Sannie Lai, RN BScN ${ }^{1,3}$

Samira Zanjani, RN BScN 1,3

Peter G. Rossos, MD MBA ${ }^{2,3}$

\author{
${ }^{1}$ Division of General Internal Medicine, University Health Network, Toronto, Canada. \\ ${ }^{2}$ Department of Medicine, University of Toronto, Toronto, Canada. \\ ${ }^{3}$ Centre for Innovation in Complex Care, University Health Network, Toronto, Canada.
}

Funding for part of this implementation was made possible through the work of a clinical alerting project funded by the Canadian Patient Safety Institute. The remainder of the funding was from the University Health Network.

Disclosure: None of the investigators have a direct personal financial interest in Research in Motion. Research in Motion did not fund this study and has not provided any funds to University Health Network to the best of our knowledge.

BACKGROUND: Communication between clinicians is hampered by the frequent difficulty in reaching the most responsible physician for a patient as well as the use of outdated methods such as numeric paging. The aim of this study was to evaluate the use of smartphones to improve communication on internal medicine wards.

METHOD: At the Toronto General Hospital, residents were provided with smartphones. To simplify reaching the most responsible resident for a patient, a smartphone designated as "Team BlackBerry" was also carried by each senior resident and then passed to the resident covering the team at night and on weekends. Nurses were able to send email messages or call smartphones directly.

RESULTS: There were on average of 9.1 incoming calls, 6.6 outgoing calls, 14.3 received emails, and 2.8 sent emails per day to each Team BlackBerry. Team BlackBerrys received up to 35 calls and 57 emails per day. Residents strongly preferred the smartphones over conventional paging with perceived improvements in all items measured and felt that it improved efficiency and communication. Although nurses perceived a reduction in the time required to contact a physician (27.6 vs. 11 minutes $P<0.001$ ), their overall satisfaction with physician's response time for urgent issues did not improve significantly.

DISCUSSION: When smartphones were used for clinical communication, residents perceived an improvement in communication with them. Residents strongly preferred emails as opposed to telephone calls as the prime method of communication. Further objective evaluation is necessary to determine if this intervention improves efficiency and more importantly, quality of care. Journal of Hospital Medicine 2010;5:553-559. ( 2010 Society of Hospital Medicine.

KEYWORDS: cellular phones, email, handheld computers, hospital communication systems, telecommunications.

The scope and importance of communication between clinicians in the delivery of health care is increasingly being recognized. ${ }^{1,2}$ Poor communication is known to be a source of inefficiency and errors within healthcare. ${ }^{3-6}$ The major issues with communication include the frequent use of interruptive communication mechanisms and the difficulty of knowing who to contact. ${ }^{2}$ Traditional paging remains the primary method to contact a physician despite being disruptive, inefficient, and predisposing to errors. ${ }^{7-10}$ The identification of the responsible physician for a patient can also be complicated with the numerous call schedules, different coverage rules, vacations, and protected academic time for residents. In 1 observational study, $25 \%$ of calls in a hospital were attempts to identify a responsible individual for a specific role. ${ }^{11}$ A recent study revealed that $14 \%$ of pages went to the wrong physician and that $47 \%$ of these errant pages warranted an urgent response. ${ }^{12}$ In our institution, the process for a nurse to identify which physician to contact regarding a patient care issue can be complex (Figure 1).

The use of email and mobile phone technology has been recommended as a method to improve communication. ${ }^{2}$ It can improve communication between clinicians by providing a method of triaging based on importance: instantly by telephone for urgent issues and less disruptively by email for nonurgent issues. There is limited literature on the use of email for improving communication between clinicians or on the use of smartphones. In a previous pilot study, we found that with minimal training, residents were able to use smartphones on general medical wards for clinical communication and that technical challenges were minimal. ${ }^{13}$ In an intensive care setting, the use of wireless emails using smartphones was perceived by staff to improve communication. ${ }^{14}$ 


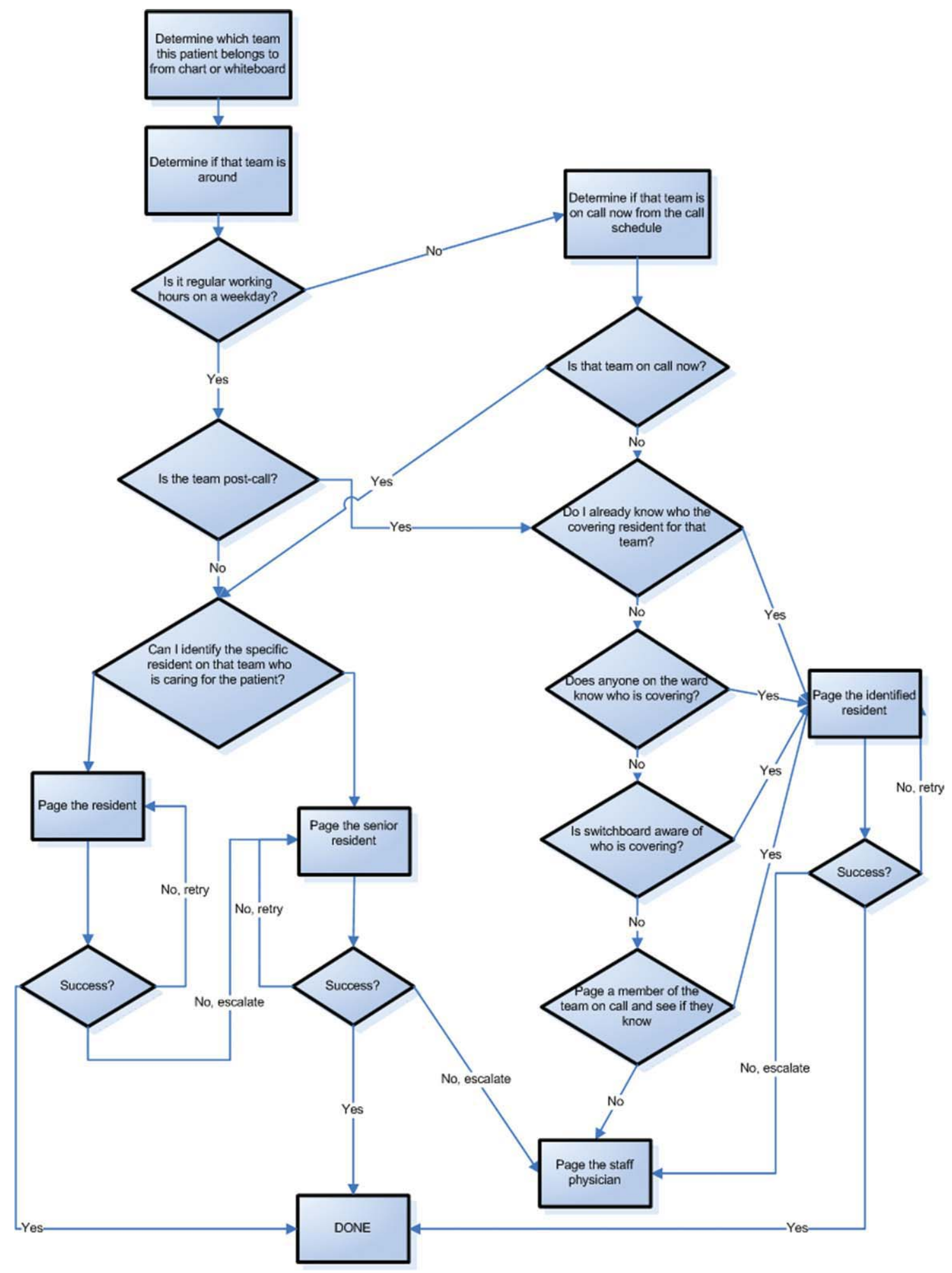

FIGURE 1. Process that nurses may need to go through to find out whom to contact regarding a care issue for their patient. [Color figure can be viewed in the online issue, which is available at wileyonlinelibrary.com.]

\section{Methods}

Aim

To evaluate the use of smartphones to improve the communication processes on an academic general internal medicine service.

\section{Setting}

We conducted the study within the General Internal Medicine service at the Toronto General Hospital, an academic teaching hospital. The service is comprised of 4 teaching teams, each with 1 staff physician, 1 senior resident, 2 to 3

2010 Society of Hospital Medicine DOI 10.1002/jhm.775

View this article online at wileyonlinelibrary.com. 
junior residents, and medical students. The environment is typically characterized by a high volume of medically complex patients and constant turnover of physicians with varying degrees of experience. Ethical review of the study was performed by the University Health Network Research Ethics Board.

\section{Program Description}

Recognizing the difficulty in implementing complex interventions within clinical care, we used a staged approach applying the standard Plan-Do-Study-Act (PDSA) methodology for quality improvement. ${ }^{15}$ This involved developing a component of the system, releasing it to the users to learn from their experience, then taking this feedback to make changes and continuing with the next cycle. This allowed early identification and quicker resolution of issues.

\section{Smartphones as Clinical Communication Tools}

The first change implemented was to supplement the standard numeric pager with smartphones as the primary communication device for the residents. The BlackBerry device (Research in Motion, Ontario, Canada) was selected because it is an efficient, usable device with voice and secure email functionality that also happens to be the standard smartphone for our hospital administrators. Beginning in March 2008, all residents on the internal medicine service were equipped with a smartphone that they kept for their entire rotation. Contact lists of all devices were prepopulated with the hospital phone numbers that were important in coordinating patient care. These included numbers such as hospital flow coordinators, radiology reporting rooms, interventional radiology, and microbiology.

To facilitate reaching the most responsible resident for patients, we provided an additional smartphone, the "Team BlackBerry," that was carried by the senior resident of each team during regular hours and then passed to the resident that was covering the team at night and on weekends. With the new process, a nurse would only require knowledge of which team the patient belonged to in order to contact the most responsible resident (Figure 2). The Team BlackBerry also received critical laboratory and medication alerts that contained clinical decision support. The laboratory and medication alerts were generated through the hospital rulebased clinical decision support system (Misys Insight).

\section{"Web-paging" to Improve Nurse-Physician Communication}

In May 2008, we implemented a platform for text paging that we called "web-paging" that allowed the nurses to efficiently send email messages securely through a hospital intranet page to the Team BlackBerry. A nurse would access the form from any computer on the wards, select the appropriate team, provide relevant information, and then send the message. Although the form would send an email, the term "web-paging" was used to create familiarity with their previous process of contacting a resident, namely paging.
This process reserved voice communication for urgent matters only while routing less urgent issues through the webpaging form. Furthermore, emails were categorized into three priority levels:

1. Call back requested messages were for issues that were not life threatening but needed immediate action or required discussion with a physician.

2. E-mail response requested messages were for issues that needed an action but could wait until the physician was available, such as cosigning an order.

3. Information only with no response necessary messages facilitated 1-way communication, such as updating the team of recent vital signs for a patient.

Physicians would receive prompt notification of a new email on their smartphone, and they could reply to the email or call back using a link within the email. Nurses were able to view the physician's email responses from an email account that was shared amongst the nurses on each ward.

\section{Program Evaluation}

The outcome measures of the study were residents' use of smartphones and perceptions of residents and nurses on the new communication process. Resident use of the smartphones was measured by the volume and the frequency of phone calls and emails over a 3-month period from September 2008 to November 2008. Residents' perceptions were measured by a survey administered prior to the start of their clinical rotation and at the end of their rotation (Table 1). Data collection for residents occurred between June 2008 and February 2009. Nurses' perceptions were measured by surveys administered to the nurses working on either day shifts or night shifts over a 1-week period prior to the intervention in March 2008 and then 6 months after in September 2008 (Table 2).

\section{Data Analysis}

We present utilization as the mean number of messages sent and received daily. We report survey responses as the mean scores on a 5-point Likert scale. For ordinal data, we described the survey responses using median and mode, and the Mann Whitney U test was used to test for differences between before and after responses. For parametric data, unpaired $t$-tests were used to look for differences in the perceptions before and after the intervention. All $P$ values are 2-sided.

\section{Results}

\section{Smartphone Usage}

Usage of emails and calls for the team devices over the three months is shown in Table 3. Most communications were through the Team BlackBerry devices with the individual devices showing less usage than the team device with 2.1 sent emails per day and 4.0 received emails per day. On the days with highest use, there were 7 calls received per 


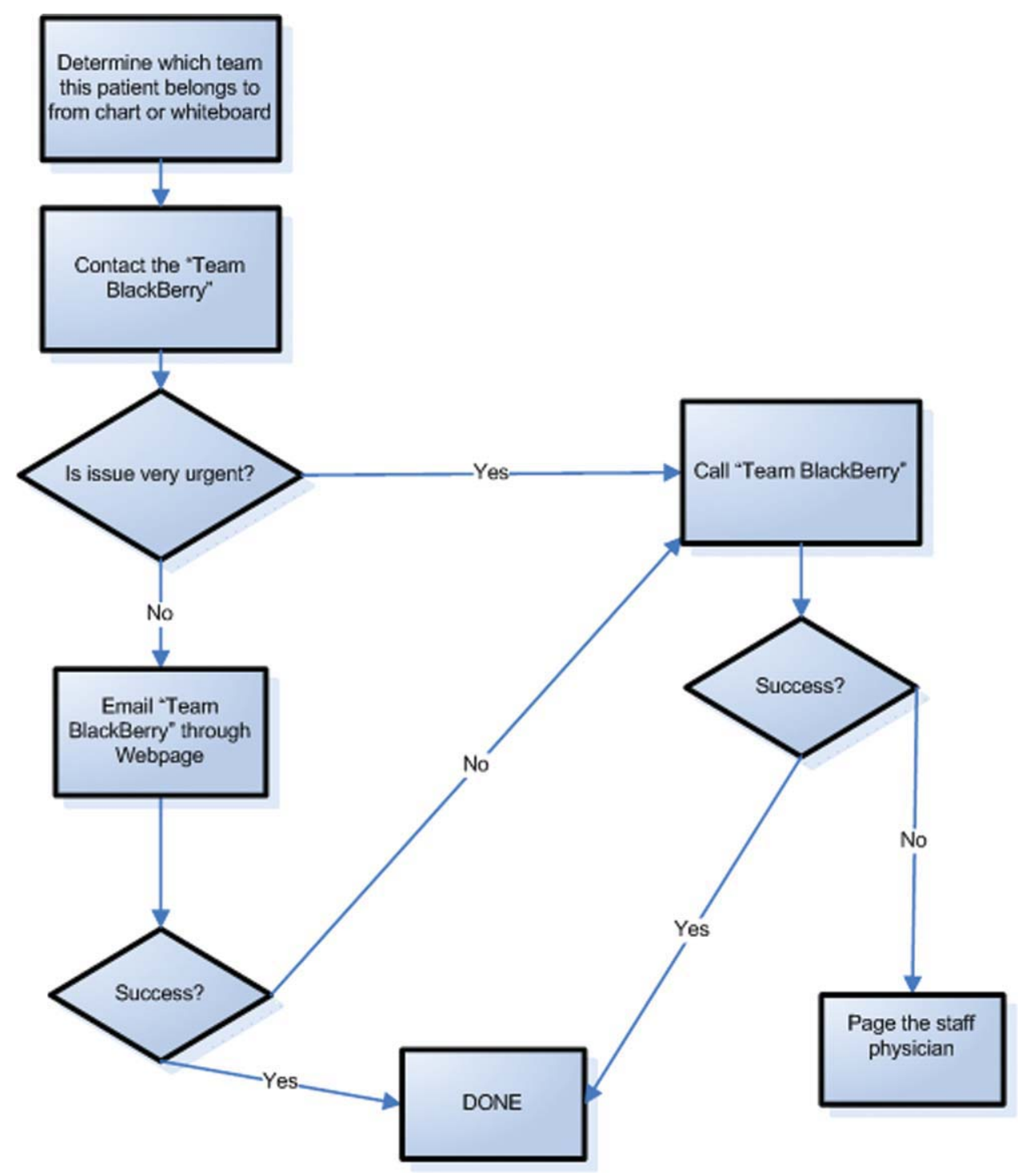

FIGURE 2. New process for nurses to contact a physician regarding a care issue for their patient. [Color figure can be viewed in the online issue, which is available at wileyonlinelibrary.com.]

hour and 6 emails received per hour at peak times through the day to a Team BlackBerry. Web-paging was the largest source of emails sent to Team BlackBerrys (42\%). Critical laboratory or medication alerts represented $17.1 \%$ and the remainder $(40.9 \%)$ were other communications such as communication from residents, staff physicians, pharmacists, and allied health professionals. Of the web-paging communications, $35.1 \%$ requested a call back, $22.6 \%$ requested email response, and $42.2 \%$ were informational items only that did not require a response.

\section{Resident Perceptions}

Of the 91 residents, 59 residents completed the presurveys (response rate 65\%) and 65 completed the postsurveys (response rate $71 \%$ ) (Table 1 ). There was a statistically significant improvement in all of the items measured. There were also 26 postsurvey comments with 22 describing posi- tive aspects and 9 describing negative aspects. Specific positive attributes of the new communication system that were mentioned were: easier to communicate with other physicians within the team $(\mathrm{n}=6)$, easier to communicate with other health care members of the team (nurses, allied health) $(n=3)$, and increased mobility $(n=2)$. The following are some examples:

"Blackberrys are great for contacting team member, able to mobile in hospital instead of waiting by phone since we're so busy..."

"Excellent for interpersonal communication. It definitely sped up patients care ie, consults, getting investigations done and discharges. Everyone should have one."

"Text messaging, especially in group format so that all members participate in conversations is the primary communication tool of choice. Emailing is a necessity of the modern workforce." 


\begin{tabular}{|c|c|c|c|}
\hline & $\begin{array}{l}\text { Pre-Survey } \\
(\mathrm{n}=59) \text { Median, } \\
\text { (mode, mean) }\end{array}$ & $\begin{array}{l}\text { Post-Survey } \\
\text { ( } \mathrm{n}=65) \text { Median, } \\
\text { (mode, mean) }\end{array}$ & $P$ value \\
\hline Postgraduate Year 1 & 39 (66.1\%) & $43(66.2 \%)$ & \\
\hline Postgraduate Year 2 & $17(28.8 \%)$ & $19(29.2 \%)$ & \\
\hline Postgraduate Year 3 & $3(5.1 \%)$ & $3(4.6 \%)$ & \\
\hline \multicolumn{4}{|c|}{ The following questions used a Likert scale with 1 representing "strongly disagree" and 5 representing "strongly agree" } \\
\hline Q1. I never have issues accessing a phone to discuss patient care issues & $3(2,2.8)$ & $4(5,3.9)$ & $<0.001$ \\
\hline Q2. I often waste a lot of time waiting for my pages to be answered & $4(4,4.1)$ & $4(4,3.3)$ & 0.004 \\
\hline Q3. Communicating with my team often takes a lot of time & $4(4,3.5)$ & $2(1,2.5)$ & $<0.001$ \\
\hline $\begin{array}{l}\text { Q4. I have quick and easy access to contact information } \\
\text { (eg, allied health, departments, consultants, etc...) necessary for providing patient care }\end{array}$ & $3(2,2.6)$ & $4(4,3.7)$ & $<0.001$ \\
\hline Q9. Overall, being phoned on the BlackBerry (instead of receiving a page) is disruptive to me & & $3(2,3.0)$ & \\
\hline Q10. I consider Internet access on the BlackBerry useful for looking up information related to patient care. & & $3(3,3.0)$ & \\
\hline Q11. I prefer not having a BlackBerry at all than having to deal with technical difficulties when I use it & & $2(1,1.9)$ & \\
\hline Q12. I feel that overall a BlackBerry saves me time & & $4(5,4.3)$ & \\
\hline
\end{tabular}

\section{TABLE 2. Nursing Perceptions on the Change in Communication Process}

\begin{tabular}{|c|c|c|c|}
\hline & $\begin{array}{l}\text { Pre-Survey } \\
(\mathrm{n}=27) \\
\text { Median, Mode }\end{array}$ & $\begin{array}{l}\text { Post-Survey } \\
(\mathbf{n}=35) \\
\text { Median, Mode }\end{array}$ & $P$ Value \\
\hline Total number of times you tried to page, email or telephone a resident or medical student in the shift just completed. & 3.70 & 2.51 & 0.16 \\
\hline Did you need to repeatedly try to contact (page, email, or call) a resident at any time during the shift regarding the same issue? & $80 \%$ & $34.3 \%$ & 0.0012 \\
\hline $\begin{array}{l}\text { Total amount of time that you spent in the last shift trying to contact doctors or other health care providers (minutes). } \\
\text { The following questions used a Likert scale with } 1 \text { representing "Strongly Disagree" and } 5 \text { representing "Strongly Agree:" }\end{array}$ & 27.6 & 11.1 & $<0.001$ \\
\hline Overall, I am satisfied with physicians' response time when I need to contact them urgently. & $3(4)$ & $4(4)$ & 0.54 \\
\hline I spend a lot of time away from the bedside just trying to contact physicians. & $3(4)$ & $3(3)$ & 0.17 \\
\hline I like being able to call and reach the doctor directly. & & $4(5)$ & \\
\hline Overall I am satisfied with the BlackBerry/email communication system. & & $4(4)$ & \\
\hline
\end{tabular}

The 2 predominant issues described were that the direct calling by nurses was disruptive $(\mathrm{n}=6)$ and voice mail was not useful $(\mathrm{n}=3)$ :

"I dislike the voicemail as it is like paging back but you also need to make a phone call to your phone list. Also we are always being interrupted which is disruptive to work but prefer BlackBerrys."

"Helpful but often disruptive such as getting calls when seeing patients."

\section{Nursing Perceptions}

From the typical staff of 94 full-time and part-time nurses, 27 of $48(56 \%)$ completed the preintervention survey, and 35 of $54(65 \%)$ completed the postintervention survey. There

\section{TABLE 3. Average Daily Communications Using the Team BlackBerry}

\begin{tabular}{lccccc} 
& \multicolumn{2}{c}{ Phone Calls } & & \multicolumn{2}{c}{ Emails } \\
\cline { 2 - 3 } & Incoming & Outgoing & & Received & Sent \\
\hline Average & 9.1 & 6.6 & 14.3 & 2.8 \\
Minimum & 0 & 0 & 0 & 0 \\
Maximum & 35 & 45 & 57 & 13 \\
Median & 8 & 4 & 13.5 & 2 \\
\hline
\end{tabular}

was a perceived significant decrease in the need to repeatedly try to contact a physician for the same issue (80\% vs. $34.3 \%, P=0.001$ ) and a significant decrease in the perceived 
amount of time spent trying to contact clinicians (27.6 minutes vs. 11.1 minutes, $P<0.001$ ) (Table 2). On 5 point Likert scales, there was no perceived improvement in communication areas such as finding out who to reach and resident response time. Nurses appreciated the ability to call the physicians directly for urgent issues (median response 4, mode of 5).

\section{Discussion}

Principal Results

In this pilot study of using smartphones and email, residents perceived that it significantly improved their efficiency. Nurses perceived a reduction in the time spent trying to communicate with clinicians.

There were significant lessons learned. First, we found that residents readily utilized smartphones and felt that it improved their efficiency. Staff physicians and residents have outlined multiple concerns over increased workload, increasing patient complexity, and longer work hours. Therefore, interventions that improve physician productivity are essential in maintaining and improving patient care. ${ }^{16}$ From residents' comments, it appears their improved efficiency is derived not only from improved communication within the medical team, but also within the interprofessional team. Efficiency was also perceived to improve from the greater mobility of being able to walk while returning a page or while waiting for a page to be returned.

Second, we found that changing the communication process was complicated. While the number of calls and emails per day appeared manageable, the peak times had very high volumes and may actually be unmanageable. By facilitating the process of reaching the responsible physician, we may have lowered the threshold for contacting the physician and thus actually increased communication volumes. It is unclear whether the end result is beneficial as improved management of patients is balanced by the increased interruptions. Further study is required to understand the effect of increasing communication on patient outcomes.

Finally, residents indicated quite strongly that they did not want telephone calls to be the primary mode of contact as they found the frequent telephone calls too disruptive. A direct call to a physician created significant disruption to workflow requiring interrupting a task to answer the call or deferring the call to later review of a voice mail message. Accessing voice mail would typically take at least a minute, much more time than the few seconds it takes to review a numeric page or a text page. Residents quickly provided feedback that this process was unacceptable and reduced their ability to deliver care effectively. Furthermore, nurses were frustrated by the added time required to leave voice messages and the poor response rate. As information within voice mails was not acted upon, nurses quickly learned to just keep calling.

Costs of implementation were significantly more than the numeric paging system. With a cost of $\$ 5 /$ month for a numeric pager, the cost of numeric pagers for 4 medical teams with 4 physicians each was $\$ 80 /$ month (all costs in Canadian dollars). From our implementation, the approximate cost of an individual smartphone was $\$ 80 /$ month while team-based devices were $\$ 160 /$ month due to much higher usage. With 4 individual smartphones and one teambased smartphone per team, costs with the new system were approximately $\$ 1,600 /$ month. While implementing smartphones creates a significantly greater expense, it may be worthwhile if improved communication leads to more efficient and higher quality care. Our current system is funded through the hospital to allow for further evaluation, and whether other hospitals provide smartphones for clinical communications likely would depend on whether they provided a cost effective alternative to numeric pagers. This would likely require some more tangible benefit than perceived improved efficiency.

\section{Comparison With Prior Work}

Similar to the study of wireless email using smartphones in the Intensive Care Unit, physicians in our study perceived an improvement in efficiency. ${ }^{14}$ Our study adds further information to the literature since we implemented on general medical wards in an academic teaching hospital. Our study is consistent with the previous studies have documented physicians' perceptions of improved performance with mobile phones. 717

\section{Limitations}

There are limitations to this study. In order to improve communication, we implemented a complex intervention with several components: (1) smartphones for residents, (2) Webpaging for nurses, and (3) a new process of identifying the most responsible physician using Team BlackBerrys. From our surveys, it is difficult to know the relative impact of each individual component. Other interventions such as teambased alphanumeric pagers may achieve similar improvements to nurses' perceptions of improved communication.

As this was a pilot study assessing new communication methods, we informally assessed perceptions using surveys which had not been validated. While the use of formally validated surveys would be more useful, we were unable to find any that specifically addressed the innovation in communication seen with smartphones. Validity issues with the instruments and the low response rates may have contributed to the incongruent result seen with nurses' surveys in that there was a perceived improvement in time to contact physicians but not in the ease of contact of physicians or the satisfaction of the communication method. This incongruent result may also reflect that in spite of the fact that the nurses were accustomed to the new system and that things may have improved, communication issues still remain and likely would benefit from further study.

It would have also been useful to determine the effect of this intervention on numeric pages sent to traditional pagers, 
which was not captured. It is also important to realize that we did not replace the pagers but instead supplemented residents with smartphones. To replace pagers may involve significant costs to upgrade reception within hospital walls to reduce areas of little or no reception. Finally, this study was completed at 1 academic health science center, and the generalizability of the findings may be limited.

\section{Future Study}

There is great opportunity to improve communication between clinicians, and the use of smartphones, email and improved identification of the most responsible physician are some methods to achieving this. Further research would be useful to determine the relative importance of each component. With smartphones, it would be useful to know whether the benefits seen were primarily due to the mobile email or having another phone available or other features. Finally, the use of web-paging and email to communicate increases the documentation of communications, but a further improvement could be providing acknowledgement to nurses that web-pages were received and read. This would close the loop on communications, ensuring that there are no lost communications and that escalation occurs promptly for urgent communications.

In summary, we implemented and evaluated a system to route and prioritize clinical communications combining the use of phone calls and secure email messaging to smartphones. Residents strongly perceived an improvement in communication with smartphones. Further objective clinical evaluation is necessary to determine if this intervention improves efficiency and more importantly, the quality of care.

\section{Acknowledgements}

The investigators retained all control over study design, data collection, analysis and interpretation, and preparation of reports.

\section{Address for correspondence and reprint requests:}

Robert C. Wu, MD, MSc, Toronto General Hospital, 14EN-222,

200 Elizabeth St, Toronto, Ontario, Canada, M5G 2C4;

Telephone: 416-340-4567; Fax: 416-595-5826;
E-mail: robert.wu@uhn.on.ca Received 11 September 2009; revision received 2 February 2010; accepted 15 March 2010.

\section{References}

1. O'Leary KJ, Liebovitz DM, Baker DW. How hospitalists spend their time: insights on efficiency and safety. J Hosp Med. 2006;1(2):88-93.

2. Coiera E. When conversation is better than computation. $J$ Am Med Inform Assoc. 2000;7(3):277-286.

3. Brennan TA, Leape LL, Laird NM, et al. Incidence of adverse events and negligence in hospitalized patients. Results of the Harvard Medical Practice Study I. N Engl J Med. 1991;324(6):370-376.

4. Woods DM, Holl JL, Angst DB, et al. Gaps in pediatric clinician communication and opportunities for improvement. J Healthc Qual. 2008;30(5): 43-54.

5. Wilson RM, Runciman WB, Gibberd RW, Harrison BT, Newby L, Hamilton JD. The quality in Australian Health Care Study. Med J Aust. 1995;163(9): 458-471.

6. Wilson RM, Runciman WB, Gibberd RW, Harrison BT, Hamilton JD. Quality in Australian Health Care Study. Med J Aust. 1996;164(12):754.

7. Soto RG, Chu LF, Goldman JM, Rampil IJ, Ruskin KJ. Communication in critical care environments: mobile telephones improve patient care. Anesth Analg. 2006;102(2):535-541.

8. Blum NJ, Lieu TA. Interrupted care. The effects of paging on pediatric resident activities. Am J Dis Child. 1992;146(7):806-808.

9. Harvey R, Jarrett PG, Peltekian KM. Patterns of paging medical interns during night calls at two teaching hospitals. CMAJ. 1994;151(3):307-311.

10. Katz MH, Schroeder SA. The sounds of the hospital. Paging patterns in three teaching hospitals. N Engl J Med. 1988;319(24):1585-1589.

11. Coiera E, Tombs V. Communication behaviours in a hospital setting: an observational study. BMJ 1998;316(7132):673-676.

12. Wong BM, Quan S, Cheung CM, et al. Why isn't anyone returning my page? Frequency and clinical importance of pages sent to the wrong physician. Arch Intern Med. 2009;169(11):1072-1073.

13. Quan S, Wu RC, Morra D, et al. Demonstrating the BlackBerry as a clinical communication tool: a pilot study conducted through the centre for innovation in complex care. Healthc Q 2008;11(4):94-98.

14. O'Connor C, Friedrich JO, Scales DC, Adhikari NK. The use of wireless email to improve healthcare team communication. J Am Med Inform Assoc. 2009;16(5):705-713.

15. Berwick DM. A primer on leading the improvement of systems. BMJ. 1996;312(7031):619-622.

16. Okie S. An elusive balance-residents' work hours and the continuity of care. N Engl J Med. 2007;356(26):2665-2667.

17. Ramesh J, Carter AO, Campbell MH, et al. Use of mobile phones by medical staff at Queen Elizabeth Hospital, Barbados: evidence for both benefit and harm. J Hosp Infect. 2008;70(2):160-165. 